\title{
The Energy and the Linear Momentum of Space-Times in General Relativity
}

\author{
Richard Schoen and Shing-Tung Yau* \\ The Institute for Advanced Study, Princeton, NJ 08540, USA
}

\begin{abstract}
We extend our previous proof of the positive mass conjecture to allow a more general asymptotic condition proposed by York. Hence we are able to prove that for an isolated physical system, the energy momentum four vector is a future timelike vector unless the system is trivial. Furthermore, we allow singularities of the type of black holes.
\end{abstract}

In our recent solution of the positive mass conjecture, we assumed that the initial data on the three-dimensional manifold is asymptotically spherical up to terms of quadratic decay. This was the classical formulation of the conjecture. However, in a recent paper, York [5] pointed out that physically it would be very desirable to push this classical formulation to a more general setting. York's condition seems to be the most general condition that may arise from an isolated physical system. In this paper, we prove the positive mass conjecture assuming merely York's condition. Our method depends on the construction of a new initial data set which verifies our previous asymptotic condition and which has approximately the same energy. Thus the positivity of the energy in the most general setting follows from the result of our previous paper.

Let us now recall York's formulation of the positive mass conjecture. An initial data set for a space-time consists of a three-dimensional manifold $N$, a positive definite metric $g_{i j}$, a symmetric tensor $h_{i j}$, a local mass density $\mu$, and a local current density $J^{i}$. The constraint equations which determine $N$ as a spacelike hypersurface in a space-time with second fundamental form $h_{i j}$ are given by

$$
\begin{aligned}
& \mu=\frac{1}{2}\left[R-\sum_{i, j} h^{i j} h_{i j}+\left(\sum_{i} h_{i}^{i}\right)^{2}\right] \\
& J^{i}=\sum_{j} \nabla_{j}\left[h^{i j}-\left(\sum_{k} h_{k}^{k}\right) g^{i j}\right]
\end{aligned}
$$

* Supported in part by an NSF grant 
where $R$ is the scalar curvature of the metric $g_{i j}$. As usual, we assume that $\mu$ and $J^{i}$ obey the local energy condition

$$
\mu \geqq\left(\sum_{i} J^{i} J_{i}\right)^{1 / 2}
$$

An initial data set will be said to be asymptotically flat if for some compact set $C, N \backslash C$ consists of a finite number of components $N_{1}, \ldots, N_{k}$ such that each $N_{\alpha}$ is diffeomorphic to the complement of a compact set in $R^{3}$. Under such diffeomorphisms, the metric tensor will be required to be written in the form

where

$$
g_{i j}=\delta_{i j}+p_{i j}
$$

and

$$
\begin{aligned}
p_{i j} & =O\left(\frac{1}{r}\right) \\
\nabla p_{i j} & =O\left(\frac{1}{r^{2}}\right)
\end{aligned}
$$

$$
\nabla \nabla p_{i j}=O\left(\frac{1}{r^{3}}\right) .
$$

The components of $h_{i j}$ and $\nabla h_{i j}$ will be required to be $O\left(r^{-2}\right)$ and $O\left(r^{-3}\right)$ respectively. Furthermore, $R$ and $\nabla R$ will be assumed $O\left(r^{-4}\right)$.

Under the above asymptotic assumptions, one defines the ADM [1] mass of each asymptotic regime $N_{\alpha}$ to be

$$
M_{\alpha}=\frac{1}{16 \pi} \int_{\infty}\left(g_{i j, j}-g_{j j, i}\right) d S_{i}
$$

where " $\infty$ " denotes the euclidean sphere $r=$ constant in the asymptotic regime $N_{\alpha}$ in the limit $r \rightarrow \infty$.

Similarly, one can define the total linear momentum $P_{\alpha}$ of each asymptotic regime $N_{\alpha}$. Together with $M_{\alpha}$, we obtain a four momentum vector. The positive mass conjecture says that this four momentum vector is a future-pointing timelike vector for each asymptotic regime unless the space time evolved by our initial data set is the Minkowski spacetime. In this letter, we demonstrate how to reduce this more general form of the positive mass conjecture to our previous theorem [3].

Since the four momentum vector is invariant under Lorentz transformations, we have only to prove that for each $\alpha, M_{\alpha}>0$ unless the spacetime is the Minkowski spacetime. In order to show this fact, we prove that, starting from an initial data set which satisfies the more general condition of York, we can create a timesymmetric initial data set satisfying our previous asymptotic condition whose energy is not more than the original energy plus an arbitrarily small amount. This will prove that for each $\alpha, M_{\alpha} \geqq 0$. When $M_{\alpha}=0$, our previous arguments [3] show that the spacetime is the Minkowski spacetime.

More precisely we shall prove the following statement: Given $\varepsilon>0$, there exists a metric $\bar{g}_{i j}$ on $N$ with zero scalar curvature so that on each asymptotic regime 
$N_{\alpha}$ we have

$$
\begin{aligned}
\bar{g}_{i, j} & =\varphi^{4} \delta_{i j} \\
\varphi & =1+\frac{\bar{M}_{\alpha}}{2 r}+O\left(r^{-2}\right),|\nabla \varphi|=O\left(r^{-2}\right),|\nabla \nabla \varphi|=O\left(r^{-3}\right)
\end{aligned}
$$

where $\bar{M}_{\alpha} \leqq M_{\alpha}+\varepsilon$.

To prove this statement we use the method of our previous paper and a conformal method which was used by Lichneriwicz and O'Murchadha-York [2]. We first note that the method of [4], which works under the more general asymptotic conditions, allows us to assume that the scalar curvature $R \equiv 0$.

By removing all the asymptotic regimes, except $N_{\alpha}$, outside a convex ball, we may assume $N$ has only one asymptotic regime $N_{\alpha}$ with mass $M=M_{\alpha}$. Note that the methods of [3] allow compact boundary $\partial N$ with positive mean curvature. We now write $g_{i j}$ on $N_{\alpha}$ in the form

$$
g_{i j}=\left(1+\frac{M}{2 r}\right)^{4} \delta_{i j}+\tilde{g}_{i j}
$$

where $\tilde{g}_{i j}=O\left(r^{-1}\right),\left|\nabla \tilde{g}_{i j}\right|=O\left(r^{-2}\right)$, and

$$
\int_{\infty}\left(\tilde{g}_{i j, j}-\tilde{g}_{j j, i}\right) d S_{i}=0 \text {. }
$$

That $g_{i j}$ can be written in this form follows easily from the assumptions and the definitions of $M_{\alpha}$. For a large number $\sigma>0$, we choose a smooth real valued function $\zeta_{\sigma}$ satisfying

$$
\begin{aligned}
\zeta_{\sigma}(r) & =\left\{\begin{array}{lll}
0 & \text { for } & r<\sigma \\
1 & \text { for } & r>2 \sigma
\end{array}\right. \\
0 & \leqq \zeta_{\sigma}(r) \leqq 1,\left|\nabla \zeta_{\sigma}\right| \leqq c_{1}(1+r)^{-1},\left|\nabla \nabla \zeta_{\sigma}\right| \leqq c_{1}\left(1+r^{2}\right)^{-1} .
\end{aligned}
$$

We then define a slightly modified metric $g_{i j}^{\sigma}$ by

$$
g_{i j}^{\sigma}=g_{i j}-\zeta_{\sigma}(r) \tilde{g}_{i j} .
$$

We clearly have on $N_{\alpha}$

$$
\left|g_{i j}^{\sigma}-\delta_{i j}\right| \leqq c_{2}(1+r)^{-1},\left|\nabla g_{i j}^{\sigma}\right| \leqq c_{2}\left(1+r^{2}\right)^{-1}
$$

We stress that the $c_{i}$ 's are independent of $\sigma$. Since $R \equiv 0$, we also have

$$
\begin{gathered}
R^{\sigma}=0 \text { for } r<\sigma, R^{\sigma}=0 \text { for } r>2 \sigma, \\
\left|R^{\sigma}\right| \leqq c_{3}\left(1+r^{3}\right)^{-1} \text { for } \sigma \leqq r \leqq 2 \sigma .
\end{gathered}
$$

in particular, we have

$$
\left(\int_{N}\left|R^{\sigma}\right|^{3 / 2} \sqrt{g^{\sigma}} d x\right)^{2 / 3} \leqq c_{4} \sigma^{-1},
$$

so for large $\sigma$ we may apply [3, Lemma 3.3] to assert the existence of a function 
$\varphi^{\sigma}>0$ satisfying

$$
\begin{aligned}
\Delta^{\sigma} \varphi^{\sigma}-\frac{1}{8} R^{\sigma} \varphi^{\sigma} & =0 \quad \text { on } N \\
\frac{\partial \varphi^{\sigma}}{\partial n} & =0 \quad \text { on } \partial N \\
\varphi^{\sigma} & =1+\frac{A^{\sigma}}{2 r}+O\left(r^{-2}\right) \quad \text { on } N_{\alpha}
\end{aligned}
$$

where

$$
A^{\sigma}=-\frac{1}{32 \pi} \int_{N} R^{\sigma} \varphi^{\sigma} \sqrt{g^{\sigma}} d x .
$$

It follows that the metric $\left(\varphi^{\sigma}\right)^{4} g_{i j}^{\sigma}$ has zero scalar curvature, and mass $M^{\sigma}=M+A^{\sigma}$. We show that $A^{\sigma} \rightarrow 0$ as $\sigma \rightarrow \infty$. In fact, if we set $v^{\sigma}=\varphi^{\sigma}-1$, we have

$$
\Delta^{\sigma} v^{\sigma}-\frac{1}{8} R^{\sigma} v^{\sigma}=\frac{1}{8} R^{\sigma}
$$

so we multiply by $v^{\sigma}$, integrate by parts as in $[3$, p. 65], and use the Sobolev inequality [3, Lemma 3.1$]$ together with (3) to get

$$
\left(\int_{N}\left|v^{\sigma}\right| \sqrt{g^{\sigma}} d x\right)^{1 / 6} \leqq c_{5}\left(\int_{N}\left|R^{\sigma}\right|^{6 / 5} \sqrt{g^{\sigma}} d x\right)^{5 / 6} .
$$

We now estimate

$$
\begin{aligned}
\left|A^{\sigma}+\frac{1}{32 \pi} \int_{N} R^{\sigma} \sqrt{g^{\sigma}} d x\right| & \leqq \frac{1}{32 \pi} \int_{N}\left|R^{\sigma}\right|\left|v^{\sigma}\right| \sqrt{g \sigma} d x \\
& \leqq \frac{1}{32 \pi}\left(\int_{N}\left|R^{\sigma}\right|^{6 / 5} \sqrt{g^{\sigma}} d x\right)^{5 / 6}\left(\int_{N}\left|v^{\sigma}\right|^{\sigma} \sqrt{g^{\sigma}} d x\right)^{1 / 6} .
\end{aligned}
$$

By (1), (2), and (4) this implies

$$
\left|A^{\sigma}+\frac{1}{32 \pi} \int_{N} R^{\sigma} \sqrt{g^{\sigma}} d x\right| \leqq c_{6} \sigma^{-1} .
$$

We see directly from (1), (2), and the definition of $R^{\sigma}$

$$
\left|\int_{N} R^{\sigma} \sqrt{g^{\sigma}} d x-\int_{\{\sigma \leqq r \leqq 2 \sigma\}} \sum_{i, j}\left(g_{i i, j j}^{\sigma}-g_{i j, i j}^{\sigma}\right) d x\right| \leqq c_{7} \sigma^{-1} .
$$

By the divergence theorem and the definition of $g_{i j}^{\sigma}$ we have

$$
\int_{\{\sigma \leqq r \leqq 2 \sigma\}} \sum_{i, j}\left(g_{i i, j j}^{\sigma}-g_{i j, i j}^{\sigma}\right) d x=\int_{S_{\sigma}} \sum_{i, j}\left(g_{i j, i}-g_{i i, j}\right) d S_{j}-16 \pi M
$$

where $S_{\sigma}=\{r=\sigma\}$. It follows directly from the definition of $M$ that

$$
\left|\int_{S_{\sigma}} \sum_{i, j}\left(g_{i j, i}-g_{i i, j}\right) d S_{j}-16 \pi M\right| \leqq c_{8} \sigma^{-1} .
$$

Combining this with (5), (6), and (7) we get

$$
\left|A^{\sigma}\right| \leqq c_{9} \sigma^{-1}
$$


from which it follows that $M^{\sigma} \leqq M+c_{9} \sigma^{-1}$. Our assertion now follows by taking $\sigma$ so large that $c_{9} \sigma^{-1} \leqq \varepsilon$, and taking $\bar{g}_{i j}=\left(\varphi^{\sigma}\right)^{4} g_{i j}$ so that $\varphi=\varphi^{\sigma}\left(1+\frac{M}{2 r}\right)$ on $N_{\alpha}$. This completes the demonstration of our assertions.

\section{References}

1. Arnowitt, R., Deser, S., Misner, C. : Phys. Rev. 118, 1100 (1960)

2. O'Murchadha, N., York, J. : Phys. Rev. D 10, 2345-2357 (1974)

3. Schoen, R., Yau, S. T. : Commun. Math. Phys. 65, 45-76 (1979)

4. Schoen, R., Yau, S. T. : Phys. Rev. Lett. 43, 1457-1459 (1979)

5. York, J. : Preprint. 1979

Communicated by A. Jaffe

Received June 23, 1980 
